\title{
Violencia de género en la pareja: aproximación comprensiva a las vivencias de niños y niñas*
}

\section{Exposure to Intimate Partner Violence: Comprehensive Approach to Children's Experiences}

\author{
Jenniffer K. Miranda Miranda ${ }^{\mathrm{a}}$ \\ Universidad de Chile, Chile \\ ORCID: http://orcid.org/0000-0002-2565-3177 \\ Jubitza Corovic Doty \\ Universidad de Chile, Chile \\ ORCID: http://orcid.org/0000-0001-7715-628X
}

a Autor de correspondencia. Correo electrónico: jmimirand@uchile.cl

Para citar este artículo: Miranda Miranda, J. K., \& Corovic, J. (2019). Violencia de género en la pareja: aproximación comprensiva a las vivencias de niños y niñas. Universitas Psychologica, 18(3), 1-14. https:// doi.org/10.11144/Javeriana.upsy18-3.vgpa

\section{RESUMEN}

Esta investigación tuvo como objetivo comprender las vivencias de niños y niñas, de 8 a 10 años, expuestos a violencia de género en la pareja, mediante tres pruebas gráficas: Dibujo de la Figura Humana, Test de la Familia y Persona Bajo la Lluvia junto con las narrativas asociadas a estas. Los/as participantes fueron usuarios/as del Centro de Salud Mental de Pudahuel, Santiago de Chile. Los niños/as expuestos/as a violencia de género en la pareja presentan dinámicas traumatogénicas, constituyéndose la exposición como una experiencia disruptiva para los/ as niños/as. El estudio adquiere relevancia, debido a la escasa literatura internacional sobre la experiencia subjetiva de los/as niños/as expuestos/ as, aporta información contextualizada a Chile y contribuye a diseñar una metodología comprensiva para la evaluación psicológica.

Palabras clave

vivencias; niños; exposición a la violencia; violencia de género en la pareja; pruebas proyectivas gráficas.

\footnotetext{
ABSTRACT

The aim of the study was to understand experiences of school children aged 8 to 10 years old exposed to intimate partner violence through graphic projective tests -Human Figure Drawing, the Test of Family and Person in Rain- and its narrative associated. The participants accessed Mental Health Center Pudahuel, Metropolitan Region, Chile. Children exposed to intimate partner violence shown traumatogenic dynamics, highlighting such exposure as a disruptive experience for children. The study becomes relevant, due to the scarce literature in Chile, provides contextual information to our country; and contributes to design a comprehensive methodology for psychological evaluation.

Keywords

experiences; children; exposure to violence; intimate partner violence; graphic projective test.
} 
Desde la década de los noventa, la violencia contra la mujer ha adquirido gran relevancia a nivel internacional (Larraín, 1994; United Nations Children's Emergency Fund [Unicef], 2006; World Health Organization [WHO], 2005), siendo reconocida como un grave problema tanto de derechos humanos como de salud pública que afecta de forma global a toda la sociedad (WHO, 2005). Diversos estudios destacan que la violencia en la pareja constituye el tipo de violencia contra la mujer con mayor magnitud a nivel internacional (Fundación Save the Children, 2011; Unicef, 2006). Al respecto, la evidencia muestra que no solo las mujeres se ven afectadas por las consecuencias de esta, sino que también tendría un efecto expansivo en el ambiente inmediato expuesto a ella, como es el caso de los hijos e hijas de las mujeres (Holt, Buckley, \& Whelan, 2008; Lira, 1998; Miranda, 2012). Esto resulta de gran importancia debido a que diversos estudios han demostrado que la mayoría de los hogares donde existe violencia de género en la pareja (VGP) son familias con hijos o hijas (Krug, Dahlberg, Mercy, Zwi, \& Lozano, 2002).

Estudios tanto nacionales como internacionales han demostrado que una gran cantidad de niños y niñas han sido expuestos a VGP (Unicef, 2006; Krug et al., 2002). A nivel global, se estima que alrededor de 275 millones de niños/as han presenciado la violencia ejercida contra su madre (Unicef, 2006). No obstante, algunos investigadores destacan que aún no es posible conocer con exactitud la magnitud de este fenómeno, debido a que durante mucho tiempo ha sido invisibilizado (Buckley, Holt, \& Whelan, 2007; Casas, Riveros, \& Vargas, 2012; Holt et al., 2008; Lizana, 2012; Servicio Nacional de la Mujer [Sernam], 2009). En cuanto a la realidad de Chile, el Servicio Nacional de Menores (Sename, 2012) indica que 5467 niños y niñas han estado expuestos a este tipo de violencia, lo que corresponde al $12.6 \%$ del total de infantes que sufren algún tipo de maltrato infantil en este país, constituyendo el tercer tipo de mayor prevalencia.
Aún no existe una conceptualización estandarizada sobre el fenómeno que implique a las hijas e hijos de parejas donde existe VGP (Miranda, 2012). Sin embargo, diversos estudios se han esforzado por visibilizar y ampliar la comprensión de esta problemática, observándose en la literatura especializada una evolución de las terminologías y tipologías propuestas (Evans, Davies, \& DiLillo, 2008; Lizana, 2012; Miranda, 2012). En la actualidad, la mayoría de investigadores coinciden en que el término "exposición" es el que captura de manera más comprensiva la realidad de este fenómeno. Al respecto, en general, los autores concuerdan en que la exposición a VGP ocurriría cuando los/as niños/as ven, oyen, se encuentran involucrados o experimentan las consecuencias de la violencia acontecida entre sus cuidadores (Evans et al., 2008). En esta línea, Holden (2003) ha propuesto una taxonomía de 10 tipos diferentes de exposición a VGP donde se incluyen situaciones en las que los menores estarían involucrados de forma más directa en la violencia (p. ej., el niño/a sufre agresiones físicas) hasta otras experiencias en las que serían aparentemente menos conscientes de esta ocurrencia (p. ej., la VGP ocurre cuando el/la niño/a no está en casa).

La literatura ha evidenciado consistentemente que los/as niños/as que viven en hogares violentos se verían afectados en su bienestar y desarrollo psicológico general (Casas et al., 2012; Edleson, 1999; García-Moreno \& Watts, 2011; Holt et al., 2008; Larraín, 1994; Margolin \& Gordis, 2004; Miranda, 2012; WHO, 2005). Diversos autores han centrado la atención en que el fenómeno de la exposición a VGP constituiría una vivencia traumática, debido a las dificultades que tienen los/as niños/as de comprender y de otorgarle un sentido y significado a lo que se encuentran experimentando (Barudy \& Dantagnan, 2005), donde la situación traumática sobrepasaría sus recursos (Barudy \& Marquebreucq, 2006). No obstante, al existir diferencias en la variabilidad y durabilidad de los síntomas que manifiestan los menores expuestos a VGP, no es posible conceptualizar a priori esta exposición de una manera traumática, siendo necesario valorar la 
interrelación de factores protectores y de riesgo que atañen al niño o niña (Corovic, 2014; Miranda, 2012).

En este sentido, James (1996 en Barudy \& Dantagnan, 2005; Lizana, 2012) destaca la relevancia clínica del Modelo Traumatogénico creado por Finkelhor y Browne (1985), que por ser comprensivo y dinámico, otorga un sentido explicativo a la sintomatología manifestada por los infantes que han sufrido agresión sexual. Más aún, James amplía su aplicación a otras situaciones que podrían dar origen al trauma ante un evento disruptivo, como es el caso de la exposición de menores a VGP. Así, a las cuatro dinámicas traumatogénicas o traumatizantes planteadas originalmente por Finkelhor y Browne (1985), como son impotencia, estigmatización, pérdida y traición, James (1996 en Barudy \& Dantagnan, 2005; Lizana, 2012) agrega las dinámicas que denomina culpa, rabia o destructividad y disociación. Esta conceptualización y sistematización facilita la comprensión del impacto generado y el desarrollo de planes de intervención para los niños y niñas expuestos a VGP (Barudy \& Dantagnan, 2005).

A nivel internacional, el reconocimiento de la magnitud e impacto que genera la exposición a VGP en los niños y niñas ha conducido a la proliferación de diversas investigaciones (Buckley et al., 2007; Edleson, 1999; Kitzmann, Gaylord, Holt, \& Kenny, 2003; Miranda, 2012; Sternberg, Baradan, Abbott, Lamb, \& Guterman, 2006; Wolfe, Crooks, Lee, McIntyre-Smith, \& Jaffe, 2003), con el fin de comprender a cabalidad este fenómeno. Junto con ello, los investigadores han focalizado sus esfuerzos en desarrollar estrategias de intervención, tanto preventivas como psicoterapéuticas, que sean pertinentes y eficaces para aumentar el bienestar integral tanto de las madres como de sus hijos e hijas. En Chile, a pesar de la alta prevalencia de menores expuestos a VGP, hay una escasez de estudios sobre esta problemática, lo cual constituye una importante limitante para el diseño de intervenciones psicológicas contextualizadas. Actualmente, las que se implementan con esta población se fundamentan en los resultados de investigaciones internacionales, en su mayoría proveniente de países anglosajones.

Por otro lado, la información recopilada en los estudios internacionales se basa predominantemente en los reportes de las madres, así como de los profesionales a cargo del tratamiento de estos niños/as (Holt et al., 2008), y se han focalizado principalmente en los aspectos sintomatológicos que conlleva la exposición a VGP (Buckley et al., 2007; Fundación Save the Children, 2011; Kitzmann et al., 2003; Lizana, 2012; Margolin \& Gordis, 2004; Osofsky, 2003; Sternberg et al., 2006; Unicef, 2006; Wolfe et al., 2003). Es por esta razón que actualmente los investigadores enfatizan la importancia de explorar las vivencias y la percepción desde primera fuente, es decir, desde la propia perspectiva de los pequeños (Holt et al., 2008; Miranda, de la Osa, Granero, \& Ezpeleta, 2011, 2013a, 2013b), siendo este el objetivo principal del presente estudio. Así, este trabajo se suma a líneas recientes de investigación que enfatizan la necesidad de incluir las perspectivas de los/as niños/as en los estudios sobre infancia, buscando levantar sus voces, promoviendo su participación activa y validando sus producciones (Miranda, Cortés, \& Vera, 2017).

Para fines de esta investigación, se entendieron las vivencias desde el enfoque teórico del constructivismo evolutivo, considerándolas como la manera como los/as niños/as dotan de sentido y significación personal su experiencia (Benyakar \& Lezica, 2005), es decir, la manera en que la realidad existe para el individuo. La vivencia se circunscribe al tiempo, así se le otorga continuidad al proceso de construcción de la experiencia, conjugando pasado, presente y futuro. Según Benyakar y Lezica, al experimentar una situación como traumática, se produce una desarticulación del afecto con la representación, impidiendo la conformación de la vivencia. Así, un evento experimentado como traumático generaría una discontinuidad y/o disrupción en la dotación de sentido por parte del individuo, interrumpiendo su comprensión.

Considerando todo lo anterior, esta investigación tuvo el propósito de aproximarse a las vivencias de los/as niños/as expuestos a 
VGP a través de dos fuentes de información, como son las pruebas proyectivas gráficas y las narrativas asociadas a ellas. La elección de estas fuentes de información se fundamentó en que los infantes que han experimentado una situación traumática, generalmente tienen dificultad para relatar lo vivenciado (Malchiodi, 2001). Por ello, una actividad lúdica, presente en la cotidianeidad de cada niño/a, podría ser una manera no invasiva de acceder a sus experiencias, pudiendo facilitar la externalización de sus emociones (Cabezas, 2007; González, 2006; Malchiodi, 2001; Opazo \& Rivera, 2009).

De esta forma, el objetivo que orienta y guía el presente estudio es comprender las vivencias de niños y niñas escolarizados de 8 a 10 años de la región metropolitana, expuestos a violencia de género en la pareja, mediante las expresiones gráficas y narrativas del Dibujo de la Figura Humana, del Test de la Familia y de la Persona Bajo la Lluvia.

\section{Método}

\section{Participantes}

La muestra estuvo compuesta por 12 niños/ as escolares, entre 8 y 10 años; 7 de sexo femenino, expuestos a VGP, pertenecientes a la comuna de Pudahuel de la Región Metropolitana y asistentes del Centro Comunitario de Salud Mental (COSAM) de la misma. Este centro tiene la función de colaborar con la comunidad para mejorar la salud mental de las personas, y por tanto, mejorar la calidad de vida en la comuna. El rango etario de 8 a 10 años, registra una alta demanda de asistencia, particularmente para proporcionar atención psicológica a niños y niñas que han estado expuestos/as a VGP a lo largo de su vida. Consistentemente, a nivel nacional, el Anuario Estadístico Institucional del año 2011 señaló que el grupo etario de mayor atención ante esta problemática corresponde al de 6 a 11 años (Sename, 2012).

El tipo de muestreo utilizado fue el abierto (Strauss \& Corbin, 2002), el cual se realizó de forma intencionada, con el fin de que los/as participantes cumplieran con ciertos criterios de inclusión con base en los objetivos propuestos. Los requisitos de inclusión para participar en el estudio fueron: 1) cumplir con el rango etario (8-10 años); 2) haber sido expuesto a la VGP a lo largo de su vida; 3) asistir al Centro Comunitario de Salud Mental; 4) que la VGP se encontrara detenida; 5) no haber realizado un proceso psicoterapéutico previo relacionado con este fenómeno; 6) estar inscritos en el taller grupal dirigido para niños y niñas expuestos a la VGP en el COSAM de Pudahuel y 7) tener firmado el asentimiento por parte de los/as niños/as y el consentimiento por parte de las madres. Los criterios de exclusión fueron: presentar diagnóstico de retraso mental, trastornos severos del desarrollo, dificultades motoras que podrían generar un impedimento en la realización de las gráficas y haber sido víctima de agresiones sexuales, lo que podría interferir en la diferenciación del análisis de los/as niños/as que solo han sido expuestos a VGP.

\section{Instrumentos}

Dibujo de la Figura Humana ([DFH]; Hammer, 2006; Koppitz, 1974; Portuondo, 1992)

Prueba proyectiva gráfica utilizada para que las personas puedan seleccionar, desde su mundo interno, las sensaciones, emociones y percepciones asociadas a su autoconcepto o imagen corporal, expresando sus necesidades y conflictos relacionados con su propio cuerpo. Este instrumento moviliza la externalización de tensiones y exigencias vitales. Además, este instrumento permitiría acceder al nivel evolutivo del niño/a (Goodenough, 1926/1951; Koppitz, 1974).

Persona Bajo la Lluvia ([PBLL]; Barilari, Beigbeder, EG Colombo, 2009; Hammer, 2006; Querol Eु Chaves, 1997)

Prueba proyectiva gráfica que permite explorar la imagen corporal del niño/a bajo condiciones 
estresantes, lo cual es representado por la lluvia, facilitando el entendimiento de cómo el individuo afronta diversas dificultades (Hammer, 2006; Querol \& Chaves, 1997). Además, al ser aplicada en conjunto con el DFH, otorgaría información adicional al comparar los resultados de ambas pruebas (Hammer, 2006). Este instrumento ha sido ampliamente utilizado en menores que han sufrido diferentes experiencias de maltrato.

Test de la Familia (Capella Eु Miranda, 2003; Corman, 1967; Hammer, 2006; Lluis-Font, 2006; Zenequelli, 1982)

Prueba proyectiva gráfica que permite movilizar contenidos de la realidad que los/as niños/as no verbalizarían directamente. También posibilita insertarse en los conflictos y dificultades, es decir, vivencias vinculadas a relaciones familiares del niño o niña. Además, revela actitudes hacia otros miembros de su familia, así como el lugar que ocupa el individuo dentro de esta (Corman, 1967; Hammer, 2006; Lluis-Font, 2006). En la literatura internacional especializada en VGP, este instrumento ha sido utilizado para apoyar y facilitar que los infantes puedan expresar sus vivencias relacionadas con la VGP (Callaghan, Alexander, Sixsmith, \& Fellin, 2015).

Narrativas (Ochs Eु Capps, 1996; White 8 Epson, 1993)

Al finalizar la realización de cada dibujo, se solicitó a los sujetos que escribieran una historia vinculada con lo graficado. Este requerimiento facilitaría el enriquecimiento de la prueba, y otorgaría mayor entendimiento en torno al área problemática (Portuondo, 1992).

\section{Procedimiento}

El estudio fue aprobado por el COSAM de Pudahuel cumpliendo con los requerimientos éticos para este tipo de investigaciones. El centro accedió a que la aplicación de los instrumentos se realizara antes de iniciar los talleres grupales dirigidos a la población en estudio. Tras obtener su autorización, se realizó la selección de la muestra mediante la revisión de fichas de pacientes inscritos a talleres grupales, identificando a aquellos que cumplían con las características y requisitos de la investigación. Se obtuvo el consentimiento informado de la figura materna y el asentimiento informado de los/as niños/as, y se aplicaron colectivamente los instrumentos seleccionados, en la siguiente secuencia: $\left.\left.1 .^{\circ}\right) \mathrm{DFH}, 2 .^{\circ}\right) \mathrm{PBLL}$ y $\left.3 .^{\circ}\right)$ Test de la Familia. Cuando los/as participantes terminaban la ejecución de cada gráfica, se solicitaba que elaboraran una historia asociada. La aplicación colectiva de las gráficas no posibilitó considerar algunos elementos expresivos, tales como la secuencia de los elementos dibujados, ni el tiempo individual de ejecución.

\section{Plan de análisis}

Se realizó un análisis de carácter mixto (cuantitativo y cualitativo), con el fin de lograr una mirada comprensiva e integral respecto a las experiencias subjetivas de los/as niños/as expuestos a VGP. En primer lugar, se puntuaron los ítems evolutivos del DFH, según el estudio normativo de Angulo y Valenzuela (2011), con el fin de registrar la presencia (1) o ausencia (0) de los ítems evolutivos esperados, según edad y sexo. Posteriormente, se puntuó la ausencia o presencia de un set de indicadores gráficos seleccionados con base en diversos estudios con población victimizada (Barilari et al., 2009; Capella \& Miranda, 2003; Colombo \& Gurvich, 2010; Corman, 1967; Hammer, 2006; Koppitz, 1974; Lluis-Font, 2006; Portuondo, 1992; Querol \& Chaves, 1997; Zenequelli, 1982). Luego, se realizó una integración de la totalidad de indicadores asociados a efectos psicológicos comúnmente registrados en niños y niñas ante la exposición a la VGP, los que fueron clasificados en una de las seis dinámicas traumatogénicas postuladas por Barudy y Dantagnan (2005) y Lizana (2012). 
Los puntajes de los indicadores seleccionados se sistematizaron usando un análisis cuantitativo descriptivo de tipo nominal mediante una distribución de frecuencias (Hernández, Fernández, \& Baptista, 2003). En los indicadores que presentaron mayor frecuencia, se examinó su asociación con las dinámicas traumatogénicas. Para complementar la información obtenida, se incluyeron observaciones cualitativas de los dibujos.

En cuanto a las narrativas, se realizó un análisis de contenido (Berelson, 1952 en Hernández et al., 2003; Fernández, 2002), donde se consideró el tema de la historia como elemento principal para la codificación, teniendo como base los conocimientos sobre los efectos psicológicos causados por la exposición a VGP en los/as niños/ as.

Finalmente, se realizó un análisis de complementariedad (Capella \& Miranda, 2003), donde se integraron tanto los resultados obtenidos en las gráficas como en las narrativas, considerando factores protectores y de riesgo en el contexto de cada menor.

\section{Resultados}

Las características de los/as participantes, en relación con su sintomatología, tipo de agresión y exposición a VGP se presentan en la Tabla 1.

\section{Tabla 1}

Características de los participantes en relación con su tipo de exposición a VGP, tipo de agresión y sintomatología

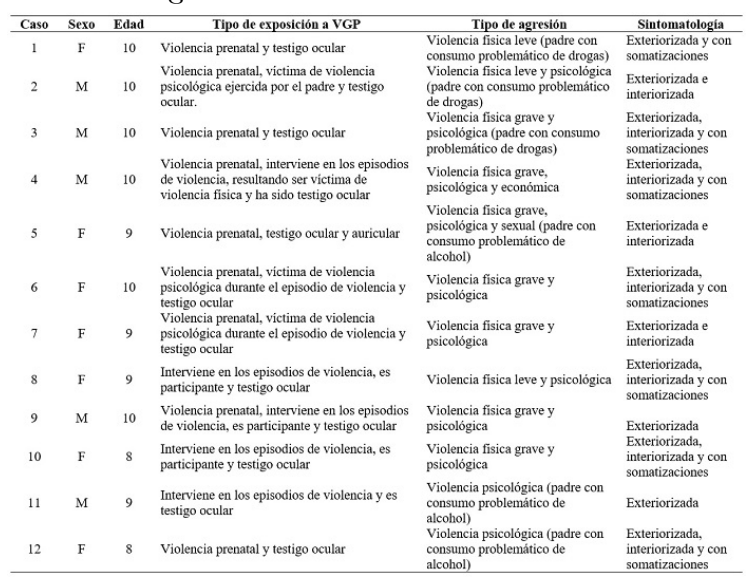

A continuación, se presenta una breve síntesis de los hallazgos sobre las vivencias de niños/ as expuestos a VGP, tras el análisis de las dos fuentes de información utilizadas en este estudio: gráficas y narrativas. Los resultados se organizan en torno a las dinámicas traumatogénicas antes mencionadas y se exponen citas textuales de los/ as participantes.

En primer lugar, se destaca que tres niños presentaron un bloqueo en la creación de narrativas, en contraste con la adecuada ejecución de las gráficas. Dichos participantes (casos 2, 3 y 4), obtuvieron un menor porcentaje en los ítems evolutivos y elaboraron historias más breves.

La dinámica de estigmatización se registró en la totalidad de los/as niños/as, en al menos una de las dos fuentes de información. Los indicadores gráficos asociados a esta dinámica son: tamaño del dibujo pequeño (indicadores expresivos en las tres pruebas proyectivas gráficas) y brazos cortos (indicadores de contenido del DFH, PBLL y Test de la Familia). Estos indicadores reflejarían sentimientos de inferioridad, concordante con las narrativas que indican una percepción de sí mismo predominantemente negativa, definida por problemas de comportamiento, así como por dificultades en su ambiente familiar, particularmente conflictos entre las figuras parentales y sentimientos de culpa.

Había una vez una familia que vivía siempre juntos y nunca se separan; una vez su hija se portó mal y la mamá y el papá pelearon porque la hija dijo que la mamá y el papá le había dicho y la hija empezó a preocuparse porque los papás nunca habían peleado en la vida y en ese momento se escapó [...]. (Caso 7, Test de la Familia)

La dinámica de impotencia que aparece en la totalidad de los participantes de este estudio, registra una percepción del mundo como amenazante y hostil, apreciándose sentimientos de indefensión, impotencia, vulnerabilidad y de incapacidad ante el afrontamiento del medio. Los indicadores gráficos relacionados con esta dinámica fueron: movimiento rígido y borraduras (indicadores expresivos de las tres pruebas 
proyectivas gráficas), omisión de la nariz, piernas pequeñas o cortas y presencia de rigidez corporal (DFH); nubes, lluvia, lluvia torrencial, rayos, omisión de nariz y presencia de rigidez corporal (PBLL) y, ojos como puntos, omisión de pies, piernas pequeñas o cortas y rigidez corporal (Test de la Familia). Además, se observó que el indicador expresivo de emplazamiento central va disminuyendo en la PBLL y Test de la Familia, de donde se podría inferir que cuando el/la niño/a se ve ante elementos que le generan tensión, la seguridad de sí mismo se aminoraría, como por ejemplo: "Había una vez una familia que era feliz y de repente sale un señor malo que convirtió a esta familia en pura maldad. Fin" (Caso 2, Test de la Familia). No obstante, en las narrativas se apreció una ambivalencia respecto a la autopercepción de vulnerabilidad ante el medio y, a su vez, autonomía para hacer frente a determinados sucesos.

La dinámica de rabia o destructividad también fue un elemento central, ya que se estuvo presente en los doce participantes de esta investigación. Los indicadores gráficos con mayor frecuencia fueron: trazos cortos y presión fuerte (indicadores expresivos en las tres pruebas proyectivas gráficas), hombros grandes (DFH); cuello grueso y hombros grandes (PBLL). Estos signos dan cuenta de sentimientos de tensión, agresividad y hostilidad hacia el medio, lo cual coincide con lo observado en las narrativas, ya que se aprecia una utilización de estrategias impulsivas en la resolución de conflictos y una visión de sí mismo negativa, con tendencia a la agresividad.

Asimismo, el análisis de las narrativas permite distinguir diversos matices en la manifestación de esta dinámica, según el género del niño/a. Por un lado, en los niños se aprecia el despliegue de conductas agresivas, como el uso de objetos destructivos para defenderse (p. ej., pistolas y cuchillos): "Érase una vez un hijo que mataba a personas y fin" (Caso 3, DFH). Por su parte, las niñas preferirían la resolución de conflictos a través de comportamientos autodestructivos, pues en las narrativas se observa la realización de conductas de riesgo que ponen en peligro su integridad física, como son el hecho de escaparse, perderse y/o enfermarse.

Otra dinámica que se presenta en ambos análisis es la pérdida o traición. Los indicadores expresivos en las tres pruebas proyectivas gráficas que representan este elemento fueron: escasez de detalles, boca cóncava u oralmente receptiva, presencia de línea media y ausencia de línea apoyo (DFH); paraguas hacia la derecha y ausencia de la línea de apoyo (PBLL) y boca cóncava u oralmente receptiva, valorización y preferencia por la figura materna, desvalorización de la figura paterna, ausencia de la línea de apoyo y padres dibujados con distancia entre sí (Test de la Familia). Estos indicadores coinciden con los contenidos que aparecen en las narrativas de tres niñas (Casos 8, 10 y 12), relacionados con la figura materna como factor protector $y$ contenedor:

Había una vez una niña y una mamá, que su mamá la cuidaba como un tesoro cuando llovía su mamá se mojaba porque hacía todo para proteger a su hija, ella agradecía lo que hacía pero ella quería proteger a su mamá. (Caso 12, PBLL)

Así también, se encontró desvalorización de la figura paterna como factor de riesgo asociado a la percepción de escasas competencias parentales, tales como: falta de contención y cuidado hacia sus hijos/as.

[...] y el hijo se estaba ahogando en el mar y el papá estaba con los pelos de puntas, y el papá casi se cae al mar porque él le tiene miedo al agua. Se fueron de la playa y el hijo fue al hospital porque tenía agua en los pulmones y fue al colegio y estuvo muy bien. Fin. (Caso 10, Test de la Familia)

En la misma línea, se asocia a la figura paterna con conductas inadecuadas que llevan al comienzo de conflictos con la figura materna, tal como se aprecia en la narración del Caso 12 en el Dibujo de la Figura Humana: "Había una vez una mamá y un papá que no estaban casados y él siempre tomaba cerveza y vino y la mamá siempre lo retaba". Todo ello, podría redundar en un daño en el ámbito de la confianza relacional. 
Otro hallazgo interesante indicó que en los casos donde ambas figuras parentales se percibían como afectivamente no disponibles, la relación fraternal emergía como una alternativa para compensar necesidades afectivas; los hermanos tenderían a sustituir al padre y podrían configurarse como una figura masculina positiva. $\mathrm{Al}$ respecto, es relevante que en el DFH y el PBLL, más de la mitad de las niñas se identificarían con una figura masculina.

Por otro lado, en cinco de participantes se encontró un significativo deseo de volver a un estado anterior, en el cual retorne la felicidad y el bienestar al hogar, lo que se habría perdido producto de los conflictos parentales: "Había una vez, una familia muy triste, un día se resolvieron todos los problemas y se quisieron unos a otros y fueron felices para siempre. Fin" (Caso 5, Test de la Familia).

Consecuentemente, la dinámica de culpa también se encuentra en ambas fuentes de información. En los dibujos, los indicadores gráficos de contenido que reflejaron esta dimensión fueron: cabeza grande (DFH), manos inconclusas y omisión de manos (PBLL), y cabeza grande y omisión de manos (Test de la Familia), donde todos los/as niños/as contarían con la presencia de al menos uno de ellos. En relación con las narrativas, la culpabilidad se observa explícitamente en tres niñas (Casos 7, 8 y 12), quienes se responsabilizan por la exposición a VGP experimentada (Casos 7 y 8), asumiendo roles inadecuados para su edad y explicitando la necesidad y autoexigencia de proteger a su madre (Caso 12): "Había una vez una niña llamada Fernanda y ella se sentía mal porque sus padres estaban separados y siempre que la niña le decía la verdad a su papá, el papá se enojaba y se mandaba condoros [esta expresión se refiere en Chile a cometer errores imperdonables] (...)" (Caso 8, DFH). Asimismo, en tres niños/ as (Casos 2, 6 y 9) se aprecia sobreexigencia y sobreadaptación en sus estrategias de resolución de conflictos y, más aún, uno de ellos exhibe conductas autodestructivas, con tendencia a la somatización.

La dinámica de disociación también fue encontrada en el análisis, pero en menor medida. En las gráficas, hay presencia de distintos indicadores que reflejan la supresión y negación de los elementos de la realidad, estos fueron: líneas que se rectangularizan (indicadores expresivos en las tres pruebas proyectivas gráficas), cuello largo (DFH) y ojos sin pupilas y cuello largo (PBLL). Si bien es notable que todos los casos presentan diversos indicadores gráficos asociados a esta dinámica, en cuatro participantes (Casos 1, 3, 4 y 7) se aprecia en sus narrativas una negación y/o supresión de las dificultades familiares experimentadas: "Había una vez una familia que era feliz y eran millonarios" (Caso 3, Test de la Familia).

\section{Discusión}

El objetivo de esta investigación fue comprender las vivencias de niños y niñas, entre 8 y 10 años, expuestos a VGP, a través de la aplicación de tres pruebas proyectivas gráficas Dibujo de la Figura Humana, Persona Bajo la Lluvia y Test de la Familia y de las narrativas asociadas a estas. A continuación, se analizan los principales resultados que dan respuesta tanto a la pregunta directriz como al objetivo planteado.

En general, el análisis de ambas fuentes de información (gráficas y narrativas) sugiere alteraciones en el desarrollo psicológico esperado para la etapa evolutiva en que se encuentran los/as niños/as, apreciándose la coexistencia de diferentes conflictos intrapsíquicos que subyacen a la sintomatología manifestada por ellos/as. En este sentido, el análisis de las vivencias da cuenta de la presencia de la totalidad de las dinámicas traumatogénicas propuestas por James (1996 en Barudy \& Dantagnan, 2005; Lizana, 2012).

En primer lugar, ante el bloqueo de tres niños en la creación de sus narrativas, se hipotetiza que la escasa fluidez en la elaboración de las historias podría asociarse a dificultades emocionales de estos niños (Edleson, 1999; García-Moreno \& Watts, 2011; Holt et al., 2008; Larraín, 1994; Margolin \& Gordis, 2004; Sernam, 2012; WHO, 2005). Esto podría ser causado por un bloqueo emocional o cierto grado de inmadurez cognitiva (Koppitz, 1974), ya que la creación de historias y 
conexión de ideas requiere habilidades cognitivas y reflexivas adecuadas para llevarlas a cabo.

Se encuentran indicadores asociados a la dinámica de la culpa en los/as niños/as que han experimentado la exposición a VGP, lo cual podría manifestarse a través de conductas compensatorias y sobreadaptadas como asumir roles no esperables a su edad. Por ejemplo, la protección a la madre con el fin de aspirar a un mayor bienestar familiar (Lizana, 2012).

Los hallazgos de este estudio muestran que la dinámica pérdida o traición también se encontraría presente en los casos analizados, la cual se expresaría en la presencia de sentimientos de miedo, preocupación y constante tristeza producto de su exposición a la VGP (Baker \& Jaffee, 2007; Edleson, 1999; Fundación Save the Children, 2011; Kitzmann et al., 2003; Lizana, 2012; Osofsky, 2003; Sternberg et al., 2006; Wolfe et al., 2003). Cabe destacar que en algunos participantes se observa una percepción ambivalente de la figura paterna, coexistiendo representaciones y sentimientos contradictorios, y apreciándose incluso que se esforzarían por mantener una cercanía afectiva con su padre.

Por otro lado, en los casos en que ambos padres no se constituirían como figuras afectivamente disponibles, se observaría una alta carencia de apoyo y contención emocional, lo que, en algunas ocasiones, se vería compensado por la relación fraternal. En este sentido, por un lado, los hermanos podrían transmitir al niño/a un sentido de pertenencia al haber experimentado una situación similar a él o ella en relación con la exposición a violencia ejercida contra la madre por parte del padre. Así también, los hermanos podrían representar un modelo de figura masculina contenedora y protectora, convirtiéndose en una persona altamente significativa para el proceso de elaboración de esta experiencia traumática para el/la niño/a.

En cuanto a la dinámica de estigmatización, es posible apreciar en los/as niños/as una dificultad por explicitar directamente la visión que tienen de ellos/as mismos/as. Al respecto, en las narrativas, se observa una autopercepción predominantemente negativa, relacionada ante todo con su comportamiento, con presencia de conductas disruptivas con preferencia hacia la autoridad y agresivas, oposicionistas y autodestructivas. La ejecución reiterada de conductas disruptivas hacia los otros podría aumentar el riesgo de exclusión de su entorno social (Baker \& Jaffee, 2007; Buckley et al., 2007; Cunningham \& Baker, 2007; Edleson, 1999; Fundación Save the Children, 2011; Holt et al., 2008; Kitzmann et al., 2003; Lizana, 2012; Osofsky, 2003; Sternberg et al., 2006; Wolfe et al., 2003).

Además, se observó, tanto en el análisis de las gráficas como en las narrativas, una percepción amenazante y hostil del mundo, lo cual genera sentimientos de indefensión, inseguridad, resignación, vulnerabilidad e impotencia hacia el ambiente (Baker \& Jaffee, 2007; Edleson, 1999; Fundación Save the Children, 2011; Kitzmann et al., 2003; Lizana, 2012; Osofsky, 2003; Sternberg et al., 2006; Wolfe et al., 2003). Esta percepción negativa del entorno estaría íntimamente relacionada con un estado de constante amenaza y tensión interna como producto de la violencia en el ambiente familiar. Dichos elementos clínicos, se encontrarían relacionados a la dinámica de impotencia.

De acuerdo a los hallazgos encontrados en esta investigación, los sentimientos de impotencia y vulnerabilidad en los/as niños/as se podrían vincular a la alta necesidad de bienestar y felicidad a nivel familiar y/o al deseo de regresar a un estado anterior donde predominaban estas características, ser parte de otro hogar, no pertenecer a su familia de origen y recuperar la confianza y la seguridad de sus padres, en especial de su figura paterna.

En relación con la dinámica de rabia o destructividad, se observó en los/as niños/as un predominio de la impulsividad en la resolución de conflictos, desplegando estrategias inadecuadas tanto en el ambiente familiar y social como hacia sí mismos/as, lo cual es concordante con lo encontrado en la literatura internacional (Baker \& Jaffee, 2007; Buckley et al., 2007; Cunningham \& Baker, 2007; Edleson, 1999; Holt et al., 2008; Lizana, 2012; Wolfe et al., 2003). 
Junto con ello, se aprecian ciertas diferencias de género en la manera de solucionar los conflictos, que se podrían asimilar al rol que ocupan las figuras parentales en el hogar y la identificación de género con cada una de ellas, ya que los niños mostraron una tendencia a validar la agresividad como un medio de afrontamiento al ambiente, mientras que las niñas asumieron un rol tendiente a la pasividad y vulnerabilidad, como las figuras maternas (Baker \& Jaffee, 2007; Cunningham \& Baker, 2007; Lizana, 2012). Esto coincide con lo señalado por Evans et al. (2008), quienes refieren que los niños presentarían mayores dificultades exteriorizadas, como lo es la agresividad, mientras que las niñas tenderían a exhibir mayores problemas interiorizados, tales como depresión, ansiedad y/ o somatizaciones. Estos planteamientos difieren con los de Kitzmann et al. (2003), Sternberg et al. (2006) y Wolfe et al. (2003), que sugieren que la violencia afecta de igual forma tanto a los niños como a las niñas.

Este significativo conflicto de género se asocia con la visión del padre, representante del sexo masculino, como figura fuerte y con autodeterminación para afrontar la adversidad, reflejando que la identificación o deseo de pertenecer al sexo masculino proporcionaría mayor seguridad en el accionar. Esta situación incide en la conformación de las relaciones interpersonales, tanto aquellas que mantienen en el presente como las que podrían establecer en un futuro. En este sentido, los menores expuestos a VGP en su vida adulta podrían replicar el patrón relacional experimentado a nivel familiar, o, por el contrario, desarrollar un patrón totalmente opuesto al vivido (Baker \& Jaffee, 2007; Cunningham \& Baker, 2007; Lizana, 2012).

En referencia a la dinámica de la disociación, se apreció que todos los participantes visualizaron al mundo como hostil y amenazante, pero no todos lograron explicitar los conflictos vivenciados en la familia. El silenciamiento de lo experimentado en el hogar, se podría deber a la necesidad de negar, suprimir y/o bloquear el daño generado por esta vivencia, hipotetizando que intentan olvidar lo vivenciado, lo cual estaría asociado a una alta carga de emotividad negativa (Lizana, 2012).

Sin embargo, los efectos generados por la exposición a la VGP dependen de la interrelación entre los factores de riesgo y los protectores presentes en el contexto de desarrollo en que crecen los pequeños, lo cual podría explicar la variabilidad y el carácter único de las vivencias y consecuencias de este fenómeno.

Así, a partir de los hallazgos obtenidos en la presente investigación, se puede entender que la exposición de niños/as a VGP se constituye como una experiencia disruptiva y traumática, que genera dificultades en la integración de las vivencias al continuo vital, lo que altera la percepción que tienen de sí mismos/as y del ambiente y su capacidad de afrontamiento.

El modelo propuesto por James (1996 citado en Barudy \& Dantagnan, 2005; Lizana, 2012) constituyó una herramienta fundamental para aproximarse a las vivencias de los/as niños/as expuestos a VGP desde su propia subjetividad, ya que permitió comprender diversos efectos cognitivos, emocionales, sociales y morales ocasionados por esta situación traumática. Por tanto, los resultados obtenidos contribuyen a la comprensión de la experiencia desde los propios menores, sus vivencias, representaciones y emocionalidad asociada. $\mathrm{Al}$ respecto, destaca que el complemento de técnicas gráficas y narrativas apunta a lograr una mirada comprensiva e integral a la subjetividad de estos infantes. Sin embargo, cabe mencionar que no se debe establecer una relación causal entre indicadores gráficos y los efectos en los/as niños/as que han sufrido de exposición a la VGP, sino que debe estar dentro de una evaluación psicológica integral que no se debe reducir a la interpretación de un solo indicador (Corman, 1967; Esquivel, Heredia, \& Lucio, 1999; Hammer, 2006; Koppitz, 1974).

Cabe señalar que los resultados de esta investigación deben ser interpretados considerando sus limitaciones. Por una parte, el diseño transversal no permite establecer relaciones causales, y por otro lado, el tamaño reducido de la muestra reduce la capacidad de generalización de los resultados. No obstante, 
según la literatura revisada, en Chile no hay estudios publicados que aborden esta relevante problemática, constituyendo este trabajo en una primera aproximación a este complejo fenómeno. En su conjunto, los resultados aportan conocimientos que podrían tener importantes implicaciones prácticas para el ámbito clínico, contribuyendo al diseño de intervenciones adecuadas y pertinentes para abordar comprensiva e integralmente los efectos asociados a la exposición a VGP. Así, aportaría al diseño de lineamientos psicoterapéuticos centrados en la superación del daño psíquico y no únicamente focalizados en la remisión de la sintomatología reportada por las madres y/o los profesionales de la salud.

Resulta fundamental que futuros estudios continúen investigando sobre la problemática que implica a los niños y niñas que han sido expuestos a VGP. Sería interesante profundizar en las características diferenciales que pueden presentar las vivencias según el sexo y distintos rangos etarios; niños y niñas con distintos tipos de exposición a VGP; menores que hayan vivenciado otras experiencias traumáticas; niñas y niños de otros países latinoamericanos. Por último, se sugiere el desarrollo de investigaciones orientadas a identificar y diferenciar tanto los factores individuales como contextuales que pueden influenciar (disminuyendo o exacerbando) los efectos asociados a este fenómeno, en tanto podrían ser cruciales para las intervenciones clínicas dentro del proceso psicoterapéutico.

\section{Agradecimientos}

Los autores desean agradecer a la Vicerrectoría de Investigación y Desarrollo, Proyecto UInicia UI-19/16, Programa de Fortalecimiento de Productividad y Continuidad en Investigación, FPCI 2017, Universidad de Chile.

\section{Referencias}

Angulo, M., \& Valenzuela, M. (2011). Estudio descriptivo-comparativo de items evolutivos e indicadores emocionales de Koppitz en el DFH, en niños de 8 a 10 años sin problemas psicológicos severos, de niveles socioeconómico medio y bajo de la Región Metropolitana de Chile (Tesis inédita). Universidad de Chile, Santiago.

Baker, L., \& Jaffee, P. (2007). Woman abuse affects our children: An educator's guide. Ottawa, CA: Ontario Women's Directorate. Recuperado de http://www.lfcc.on.ca/Educ ators_Guide_to_Woman_Abuse.pdf

Barilari, Z., Beigbeder, C., \& Colombo, R. (2009). Abuso y maltrato infantil. Indicadores en 'Persona bajo la lluvia' (2.' ed.). Buenos Aires: Editorial Cauquén.

Barudy, J., \& Dantagnan, M. (2005). Los buenos tratos en la infancia. Parentalidad, apego y resiliencia. Barcelona: Gedisa.

Barudy, J., \& Marquebreucq, A. (2006). Hijas $e$ hijos de madres resilientes. Traumas infantiles en situaciones extremas: violencia de género, guerra, genocidio, persecución y exilio. Barcelona: Granica.

Benyakar, M., \& Lezica, A. (2005). Lo traumático: clínica y paradoja (Vol. 1: El proceso traumático). Buenos Aires: Biblos.

Buckley, H., Holt, S., \& Whelan, S. (2007). Listen to me! Children's experiences of domestic violence. Child Abuse Review, 16(5), 296-310. https://doi.org/10.1002/car .995

Cabezas, C. (2007). Análisis y características del dibujo infantil. Jaén, ES: Íttakus.

Capella, C., \& Miranda, J. K. (2003). Diseño, implementación y evaluación piloto de una intervención psicoterapéutica grupal para niñas víctimas de abuso sexual (Tesis inédita). Universidad de Chile, Santiago.

Callaghan, J., Alexander, J., Sixsmith, J., \& Fellin, L. (2015). Beyond "witnessing": Children's experiences of coercive control in domestic violence and abuse. Journal of Interpersonal Violence, 33(10), 1-31. https://doi.org/10.11 77/0886260515618946

Casas, L., Riveros, F., \& Vargas, M. (2012). Violencia de género y la administración de justicia. Santiago de Chile: Servicio 
Nacional de la Mujer. Recuperado de http: //estudios.sernam.cl/?m=e\&i $=185$

Colombo, R., \& Gurvich, M. (2010). Evaluación psicológica. Actualización de la prueba gráfica HTP. Buenos Aires: Editorial Cauquén.

Corman, L. (1967). El test del dibujo de la familia. Buenos Aires: Kapelusz.

Corovic, J. (2014). Estudio exploratorio descriptivo de las vivencias representadas en el Dibujo de la Figura Humana, Persona Bajo la Lluvia y Test de la Familia, en niños y niñas de 8 a 10 años expuestos a violencia de género en la pareja (Tesis inédita). Universidad de Chile, Santiago de Chile.

Cunningham, A., \& Baker, L. (2007). Little eyes, little ears. How violence against a mother shapes children as they grow. Ottawa, CA: Ontario Women's Directorate. Recuperado de http://www.lfcc.on.ca/little_eyes_little_ ears.pdf

Edleson, J. (1999). Children's witnessing of adult domestic violence. Journal of Interpersonal Violence, 14(8), 839-870. https://doi.org/10 $.1177 / 088626099014008004$

Esquivel, F., Heredia, C., \& Lucio, E. (1999). Psicodiagnóstico clínico del niño. México: El Manual Moderno.

Evans, S., Davies, C., \& DiLillo, D. (2008). Exposure to domestic violence: A metaanalysis of child and adolescent outcomes. Aggresion and Violent Behavior, 13, 131-140. https://doi.org/10.1016/j.avb.2008.02.005

Fernández, F. (2002). El análisis de contenido como ayuda metodológica para la investigación. Ciencias sociales, 96(2), 35-53. Recuperado de https://www.revistacienciassociales.ucr. ac.cr/images/revistas/RCS96/03.pdf

Finkelhor, D., \& Browne, A. (1985). The traumatic impact of child sexual abuse: A conceptualization. American Journal of Orthopsychiatry, 55(4), 530-541. https://doi .org/10.1111/j.1939-0025.1985.tb02703.x

Fundación Save the Children. (2011). En la violencia de género no hay una sola víctima (Informe). Madrid: Autor. Recuperado de https://www.savethechildren.es/sites/def ault/files/imce/docs/violencia_de_genero victima.pdf

García-Moreno, C., \& Watts, C. (2011). Violence against women: An urgent public health priority. Bulletin of the World Health Organization, 89, 1-80. https://doi.org/10.24 71/BLT.10.085217

González, R. (2006). Análisis del dibujo infantil. Revista Digital. Investigación y Educación, 23, 1-13. Recuperado de http://www.csicsif.es/andalucia/modules /mod_sevilla/archivos/revistaense/n23/230 40118.pdf

Goodenough, F. (1951). Test de inteligencia infantil por medio del dibujo de la figura humana. Buenos Aires: Paidós.

Hammer, E. (2006). Test proyectivos gráficos (2.a ed.). Buenos Aires: Paidós.

Hernández, R., Fernández, C., \& Baptista, P. (2003). Metodología de la investigación (3. ed.). México: McGraw Hill.

Holden, G. (2003). Children exposed to violence to domestic violence and child abuse: Terminology and taxonomy. Clinical Child and Family Psychological Review, 6(3), 151-160. https://doi.org/10.1023/A:102490 6315255

Holt, S., Buckley, H., \& Whelan, S. (2008). The impact of exposure to domestic violence on children and young people: A review of the literature. Child Abuse $\mathbb{E}$ Neglect, 32 (8), 797-810. https://doi.org/10.1016/j.chiabu.2 008.02 .004

Kitzmann, K. M., Gaylord, N. K., Holt, A. R., \& Kenny, E. D. (2003). Child witnesses to domestic violence: A meta-analytic review. Journal of Consulting and Clinical Psychology, 71, 339-352. https://doi.org/10.1037/0022006X.71.2.339

Koppitz, E. (1974). El dibujo de la figura humana: evaluación psicológica. Buenos Aires: Editorial Guadalupe.

Krug, E. G., Dahlberg, L., Mercy, J., Zwi, A., \& Lozano, R. (Eds.). (2002). World report on violence and health. Ginebra: World Health Organization, 2002. Recuperado de https://www.who.int/violence_injury_pr 
evention/violence/world_report/en/full_en .pdf

Larraín, S. (1994). Violencia puertas adentro: la mujer golpeada. Santiago: Editorial Universitaria.

Lira, G. (1998). Aspectos generales de la problemática del maltrato infantil: definiciones e incidencia. En Maltrato Infantil: elementos básicos para su comprensión (pp. 14-27). Villa del Mar, $\mathrm{CH}$ : Centro de promoción y apoyo a la infancia 'Paicabí'. Recuperado de https://www.academia.edu/8676180/ MALTRATO_INFANTIL_Elementos_B $\%$ C3\%A1sicos_para_su_Comprensi $\% \mathrm{C} 3 \% \mathrm{~B} 3 \mathrm{n}$

Lizana, R. (2012). A mí también me duele. Niños y niñas víctimas de violencia de género en la pareja. Barcelona: Gedisa.

Lluis-Font, J. (2006). Test de la familia. Cuantificación y análisis de variables socioculturales y de estructura familiar. Barcelona: Editorial Davinci Continental.

Malchiodi, C. (2001). Using drawing as intervention with traumatized children. Trauma and Loss: Research and Interventions, 1(1), 14-21. Recuperado de http://assets1.s impleclickcms.com/500051/tlcv1n1.pdf

Margolin, G., \& Gordis, E. B. (2004). Children's exposure to violence in the family and community. Current Directions in Psychological Science, 13, 152-155. https://d oi.org/10.1111/j.0963-7214.2004.00296.x

Miranda, J. K. (2012). Experiencias maternas de violencia: psicopatología y deterioro funcional en niñas, niños $y$ adolescentes (Tesis doctoral inédita). Universidad Autónoma de Barcelona, Barcelona.

Miranda, J. K., Cortés, C., \& Vera, J. (2017). Infancia, palabra y silencio: aproximación desde una perspectiva constructivista. Psicoperspectivas, 16(1), 91-94. https://doi.org/10.5027/psicoperspec tivas-Vol16-Issue1-fulltext-816

Miranda, J. K., de la Osa, N., Granero, R., \& Ezpeleta, L. (2011). Maternal experiences of childhood abuse and intimate partner violence: Psychopathology and functional impairment in clinical children and adolescents. Child Abuse EF Neglect, 35, 700-711. https://doi.org/10.1016/j.chiabu.2 011.05 .008

Miranda, J. K., de la Osa, N., Granero, R., \& Ezpeleta, L. (2013a). Maternal childhood abuse, intimate partner violence and child psychopathology: The mediator role of mothers' mental health. Violence Against Women, 19(1), 50-68. https://doi.org/10.11 $77 / 0886260513488686$

Miranda, J. K., de la Osa, N., Granero, R \& Ezpeleta, L. (2013b). Multiple mediators of the relationships among maternal childhood abuse, intimate partner violence, and offspring psychopathology. Journal of Interpersonal Violence, 28(14), 2941-2965. h ttps://doi.org/10.1177/0886260513488686

Ochs, E., \& Capps, L. (1996). Narrating the self. Annual Review Anthropology, 25(19), 19-43. https://doi.org/10.1146/annurev.ant hro.25.1.19

Osofsky, J. (2003). Prevalence of children's exposure to domestic violence and child maltreatment: Implications for prevention and intervention. Clinical Child and Family Psychology Review, 6, 161-170. https://doi.o rg/10.1023/A:1024958332093

Portuondo, J. (1992). La Figura Humana: test proyectivo de Karen Machover (6. ${ }^{\mathrm{a}}$ ed.). Madrid: Biblioteca Nueva.

Querol, S., \& Chaves M. (1997). Adaptación y aplicación del Test de la Persona Bajo la Lluvia. Buenos Aires: JVE Psique.

Servicio Nacional de la Mujer. (2009). Análisis y evaluación de la ruta crítica en mujeres afectadas por violencia en la relación de pareja (Documento de Trabajo n. ${ }^{\circ}$ 107). Santiago de Chile: Autor. Recuperado de http://estu dios.sernam.cl $/ \mathrm{m}=\mathrm{e} \& \mathrm{i}=79$

Servicio Nacional de Menores. (2012). Anuario Estadístico Institucional. Año 2011. Santiago de Chile: Autor. Recuperado de http://www.sename.cl/wsename/otros/2 012/anuario_2011.pdf

Sternberg, K., Baradan, L., Abbott, C., Lamb, M., $\&$ Guterman, E. (2006). Type of violence age, and gender differences in the effects 
of family violence on children's behavior problems: A mega-analysis. Developmental Review, 26(1), 89-112. https://doi.org/10.10 16/j.dr.2005.12.001

Strauss, A., \& Corbin, J. (2002). Bases de la investigación cualitativa. Técnicas y procedimientos para desarrollar la teoría fundamentada (Trad. E. Zimmerman). Medellín, CO: Universidad de Antioquia.

United Nations Children's Emergency Fund. (2006). Behind closed doors. The impact of domestic violence on children (Informe). Nueva York: Autor. Recuperado de http://www.unicef.org/media/files/Behin dClosedDoors.pdf

White, M., \& Epston, D. (1993). Medios narrativos para fines terapéuticos. Barcelona: Paidós.

Wolfe, D., Crooks, C., Lee, V., McIntyre-Smith, A., \& Jaffe, P. (2003). The effects of children's exposure to domestic violence: A meta-analysis and critique. Clinical Child and Family Psychology Review, 6(3), 171-187. https://doi.org/10.1023/A:102491 0416

World Health Organization. (2005). WHO multicountry study on women's health and domestic violence against women. Initial results on prevalence, health outcomes and women's responses. Ginebra: Autor. Recuperado de https://www.who.int/reproductivehealt h/publications/violence/24159358X/en/

Zenequelli, E. (1982). Test de la familia. Buenos Aires: Ediciones Tres Tiempos.

\section{Notas}

* Artículo de investigación. 